\title{
59 NOVEL ADAPTIVE CONTROL OF MECHANICAL SYSTEMS DRIVEN BY ELECTROMECHANICAL HYDRAULIC DRIVES
}

\author{
János F. Bitó, József K. Tar, Imre J. Rudas \\ Budapest Polytechnic \\ H-1081 Budapest, Népszínház utca 8, Hungary \\ jbito@zeus.banki.hu,jktar@donat.banki.hu,rudas@zeus.banki.hu
}

\begin{abstract}
Electric servo systems have superior performance in comparison with hydraulic drives in the small and medium applications they are comparatively expensive and may be dissipation-sensitive. In contrast to the DC motor driven systems hydraulic differential cylinders are very intricate, non-linear, strongly coupled multivariable electromechanical engines of significant parameters, which are very difficult to control or even to measure. Traditional controllers require a relatively accurate information on these parameters. In this paper a novel adaptive control is presented which evades the problem of the incomplete and inaccurate information of these parameters as well as their timedependence. The method is promising in other fields of application where the behavior of a complex system under unknown environmental interaction is to be predicted
\end{abstract}

\section{INTRODUCTION}

In spite of the definite virtues of electric DC drives, due to several practical reasons alternative solutions are widely searched, e.g. the possibility of developing pneumatic servo systems (e.g. Drakunov et al, 1997). Traditional PID control of robots driven by hydraulic differential cylinders have to cope with the problem of instabilities, too. The desirable values of the appropriate feedback parameters may significantly depend on the actual pose of the robot arm. Two different control approaches were recently proposed for such robots (Bröcker and Lemmen, 2001). One of them is based on disturbance rejection, the other one on the partial flatness principle, respectively. In each case it is necessary to measure the disturbance force and its time-derivative as well as to know the exact model of the hydraulic cylinder.

However, it is very difficult to keep these parameters under perfect control or to measure them in real-time. The viscosity of the hydraulic oil is very sensitive to the temperature normally increasing due to its circulation in the pipe system. Oil compressibility depends on the amount of air or other gases solved in it, therefore it also is temperature-dependent. Other phenomena related to friction as the adhesion of the piston at the cylinder introduce rough non-linearity into the behavior of the system. Combined with other dynamical properties of the physical system to be 
driven by such drives as well as with not always measurable external disturbances normally very complex control tasks may arise.

In general it seems to be expedient to apply adaptive control instead of trying to measure the ample set of unknown and time-varying parameters. On the other hand this adaptive control need not to be too intricate, actually shouldn't be much more complicated than an industrial PID controller. Normally, their improvement via the traditional self-tuning essentially remains within the realm of linear control and doesn't anticipate too much success. A particular branch of soft computing has recently been proposed (e.g. Tar and Rontó, 2000). In the development of the method at first certain symmetry properties of Classical Mechanics were taken into account, later the Modified Renormalization Transformation was applied for SISO systems, finally satisfactory convergence criteria were given to its extension to MIMO systems (Pátkai et al, 2002). In the meantime the method was further simplified by the introduction of the Partially Stretched Orthogonal Transformations (El Hini, 2001).

The above cited methods were tested in the control of DC motor driven electromechanical systems. In the present paper their applicability is illustrated in the case of hydraulic differential cylinders under external perturbation unknown by the controller. Modeling errors and external perturbations reveal themselves in the actual behavior of the system in comparison with that of a very simple and approximate model.

\section{OPERATION OF THE DIFFERENTIAL HYDRAULIC CYLINDER}

The operation of the differential hydraulic cylinder is described in details by Bröcker and Lemmen (Bröcker and Lemmen 2001). Let $x$ denote the linear position of the piston in $\mathrm{m}$ units. The acceleration of the piston is described by (1) as

$$
\ddot{x}=\frac{1}{m}\left[\left(p_{A}-\frac{1}{\varphi} p_{B}\right) A_{A}-F_{f}(\dot{x})-F_{d}\right]
$$

in which $p_{A}$ and $p_{B}$ denotes the pressures in chamber $\mathrm{A}$ and $\mathrm{B}$ of the piston in bar, $\varphi=A_{A} / A_{B}$, that is the ratio of the "active" surfaces of the appropriate sides of the piston, $m$ is the mass of the piston in $\mathrm{kg}, F_{f}$ denotes the internal friction between the piston and the cylinder, $F_{d}$ denotes the external disturbance forces. The pressure of the oil in the chambers also depends on the piston position as

$$
\begin{aligned}
& \dot{p}_{A}=\frac{E_{\text {oil }}}{V_{A}(x)}\left(-A_{A} \dot{x}+B_{v} K_{v} a_{1}\left(p_{A}, U\right) U\right) \\
& \dot{p}_{B}=\frac{E_{\text {oil }}}{V_{B}(x)}\left(\frac{A_{A}}{\varphi} \dot{x}-B_{v} K_{v} a_{2}\left(p_{B}, U\right) U\right)
\end{aligned}
$$

where $B_{v}$ denotes the flow resistance, $K_{v}$ is the valve amplification, $U$ is the normalized valve voltage. $E_{o i l}$ means the oil's compressibility. The oil volume in the pipes and the chambers are expressed as 


$$
\begin{aligned}
& V_{A}(x)=V_{\text {pipeA }}+A_{A} x, \\
& V_{B}(x)=V_{\text {pipeB }}+A_{B}(H-x)
\end{aligned}
$$

( $\mathrm{H}$ is the cylinder stroke.) The hydraulic drive has two stabilized pressure values, the pump pressure $p_{0}$, and the tank pressure $p_{t}$. Under normal operating conditions (that is when no shock waves travel in the pipe line) these pressures set the upper and the lower bound to $p_{A}$ and $p_{B}$. The functions $a_{1}$ and $a_{2}$ are defined in (5).

$$
\begin{aligned}
& a_{1}\left(p_{A}, U\right)=\left\{\begin{array}{l}
\operatorname{sign}\left(p_{0}-p_{A}\right) \sqrt{\left|p_{0}-p_{A}\right|} \\
\text { if } \quad U \geq 0, \\
\operatorname{sign}\left(p_{A}-p_{t}\right) \sqrt{\left|p_{A}-p_{I}\right|} \\
\text { if } \quad U<0
\end{array}\right. \\
& a_{2}\left(p_{B}, U\right)= \begin{cases}\operatorname{sign}\left(p_{B}-p_{1}\right) \sqrt{\left|p_{B}-p_{I}\right|} \\
\text { if } & U \geq 0, \\
\operatorname{sign}\left(p_{0}-p_{B}\right) & \sqrt{\left|p_{0}-p_{B}\right|} \\
\text { if } & U<0\end{cases}
\end{aligned}
$$

Under "normal conditions" $\operatorname{sign}\left(a_{1}\right) \geq 0$, and $\operatorname{sign}\left(a_{2}\right) \geq 0$, too, according to the limiting role of the pump and tank pressures. The disturbance rejection approach (Bröcker and Lemmen 2001) is based on creating the time-derivative of (1) resulting in the appearance of the time-derivative of the disturbance force in the equations. In the sequel a control approach not needing measurement of the disturbance force and exact knowledge of the system parameters will be presented.

\section{THE ADAPTIVE CONTROL APPROACH}

Supposing the need for a desired piston acceleration determined via kinematic considerations, on the basis of the available system model a desired value can be prescribed to $\left(p_{A}-p_{B} / \varphi\right)$. (In this model the piston friction and disturbance force can be omitted.) If at least $p_{A}, p_{B}, x$, and $\mathrm{d} p_{A} / \mathrm{d} t, \mathrm{~d} p_{B} / \mathrm{d} t, \mathrm{~d} x / \mathrm{d} t$ are measurable in real-time it is possible to know the actual value of this quantity and its time-derivative. Therefore a desired time-derivative can be prescribed to this quantity. By using the approximate system model via combination (2) and (3) an appropriate control signal $U$ can be proposed for this purpose. (Taking into account the inconvenient behavior of the piston's friction, it is expedient to apply a PI-type controller for $\left(p_{A}-p_{B} / \varphi\right)$, and for the desired trajectory tracking.) The adaptive controller compares with each other the realized and the desired value of $y \equiv \mathrm{d}\left(p_{A}-p_{B} / \varphi\right) / \mathrm{d} t$. Via the application of a dummy parameter $D$ of no direct physical interpretation either a symplectic matrix or a two-dimensional vector is created as 


$$
\mathbf{S} \equiv\left[\begin{array}{cccc}
0 & 0 & -y / s & D / s \\
0 & 0 & -D / s & -y / s \\
y & -D & 0 & 0 \\
D & y & 0 & 0
\end{array}\right], s=y^{2}+D^{2}, \mathbf{v} \equiv\left[\begin{array}{c}
y \\
D
\end{array}\right]
$$

This matrix/vector can be constructed for the desired and the actual value of $y$. Taking into account the definition and the group properties of the symplectic matrices it is very easy to construct a symplectic matrix $\mathbf{T}$, which transforms the actual value into the desired one as

$$
\mathfrak{I} \equiv\left[\begin{array}{c|c}
\mathbf{0} & -\mathbf{I} \\
\hline \mathbf{I} & \mathbf{0}
\end{array}\right], \quad \mathbf{S}^{T} \mathfrak{I S}=\mathfrak{I}, \quad \mathbf{S}^{-1}=\mathfrak{I}^{T} \mathbf{S}^{T} \mathfrak{I}, \quad \mathbf{T}=[\text { Des }][\text { Act }]^{-1}
$$

or, following El Hini, construct an orthogonal matrix rotating the actual vector $\mathbf{v}$ into the desired one and leaves their orthogonal sub-spaces unchanged and making the appropriate shrink/dilatation only in the direction of the transformed actual vector (this means the creation of the appropriate Partially Stretched Orthogonal Transformation). Each mapping can be created in a computationally very inexpensive manner, and they can be regarded as a kind of experimental identification of the actual system on the basis of its rough model and experimentally observed behavior.

From purely mathematical point of view this kind of system-identification can be formulated as follows: there is given some imperfect model of the system on the basis of which some excitation is calculated for a desired input $\mathbf{i}^{\mathbf{d}}$ as $\mathbf{e}=\boldsymbol{\varphi}\left(\mathbf{i}^{\mathrm{d}}\right)$. The system has its inverse dynamics described by the unknown function $\mathrm{i}^{\mathrm{r}}=\psi\left(\varphi\left(\mathbf{i}^{\mathrm{d}}\right), \mathbf{p}\right)=$ $\mathbf{f}\left(\mathbf{i}^{\mathbf{d}}\right)$ and resulting in a realized $\mathbf{i}^{r}$ instead of the desired one, $\mathbf{i}^{\mathbf{d}}$. The parameter vector p symbolizes the parameters of the actual system not taken into account in its rough model, as well as the unknown external perturbations. They may be slowly varying function of time, too. Normally only the "net" function $\mathbf{f}()$ can be observed, but there is no possibility to directly "manipulate" it. [Only the nature of the model function $\varphi()$ can be modified.] It is rather possible only to deform its actual input $\mathbf{i}^{\mathbf{d}^{*}}$ in order to achieve and maintain the $\mathbf{i}^{\mathbf{d}}=\mathbf{f}\left(\mathbf{i}^{\mathbf{4}}\right)$ situation. The Modified Renormalization Algorithm invented by Tar (e.g. in Pátkai et al, 2002) consists in creating a series of linear transformations defined as follows

$$
\begin{gathered}
\mathbf{i}_{0} ; \quad \mathbf{S}_{1} \mathbf{f}\left(\mathbf{i}_{0}\right)=\mathbf{i}_{0} ; \quad \mathbf{i}_{1}=\mathbf{S}_{1} \mathbf{i}_{0} ; \ldots ; \mathbf{S}_{\mathrm{n}} \mathbf{f}\left(\mathbf{i}_{n-1}\right)=\mathbf{i}_{0} ; \\
\mathbf{i}_{n+1}=\mathbf{S}_{n+1} \mathbf{i}_{n} ; \mathbf{S}_{\mathrm{n}} \underset{n \rightarrow \infty}{\longrightarrow} \mathbf{I}
\end{gathered}
$$

It was proved by Tar that if $\mathbf{f}()$ is flat, that is has a small gradient according to its variables, and that if the $\mathbf{S}_{n}$ matrices are close to the identity operator, the above algorithm converges to the proper deformation of the desired input. The dummy parameter $D$ has the following role: in general it avoids the occurrence of the identification from two near-zero vectors, and in particular if $|y|<<D$, the appropriate transformations will be very close to the identity operator, which is needed for the proper convergence. For guaranteeing the other condition especially 
at the beginning of the identification a simple linear transformation is applied depending on the estimated "extent" of the necessary transformation as

$$
\xi=\frac{\left\|\mathbf{i}^{d}-\mathbf{f}\right\|}{1+\max \left(\left\|\mathbf{i}^{d}\right\|,\|\mathbf{f}\|\right)}, \quad \lambda=\lambda(\xi), \quad \hat{\mathbf{i}}^{\mathrm{d}}=\mathbf{f}+\lambda\left(\mathbf{i}^{\mathrm{d}}-\mathbf{f}\right)
$$

and the original input $\mathbf{i}^{d}$ is replaced by $\hat{\mathbf{i}}^{d}$. The function $\lambda(\xi) \rightarrow 1$ if $\xi \rightarrow 0$ and it can be constructed in many various ways via applying certain parameters in it.

\section{SIMULATON RESULTS}

In the simulations $20 \%$ error was applied in modeling oil compressibility. The piston's friction was taken into account in simulating the real system only, but it was unknown for the controller. The disturbance force had an exponentially damped sinusoidal component of the initial amplitude of $200 \mathrm{~N}$ and a constant component of $500 \mathrm{~N}$.
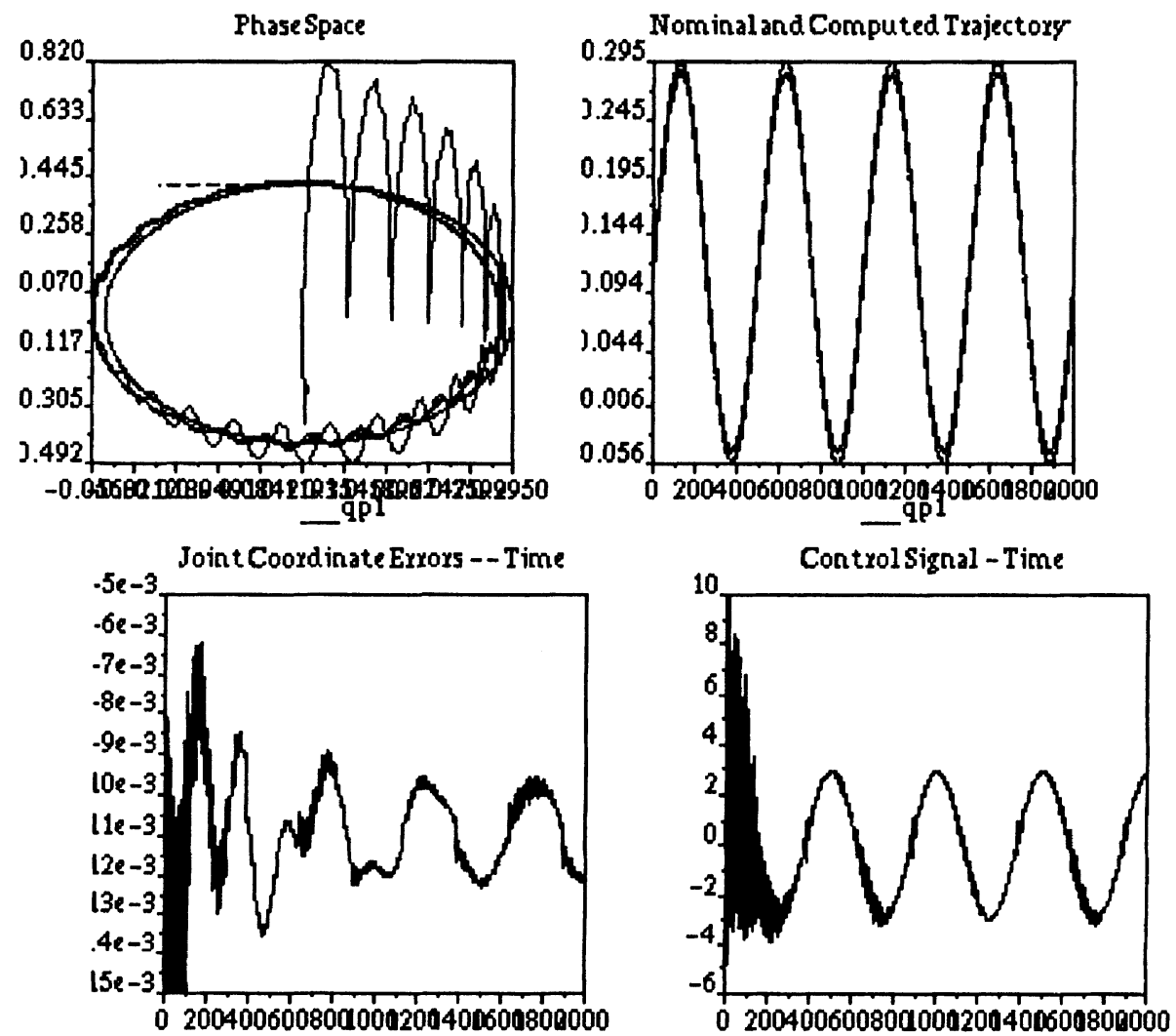

Figure 1-The phase space [ $\mathrm{m}$ and $\mathrm{m} / \mathrm{s}$ units], the nominal and the simulated trajectory [coordinate in $m$, and time in $5 \mathrm{~ms}$ units], the trajectory reproduction error versus time [in $m$ ], and the not control signal of the valve (not normalized) 

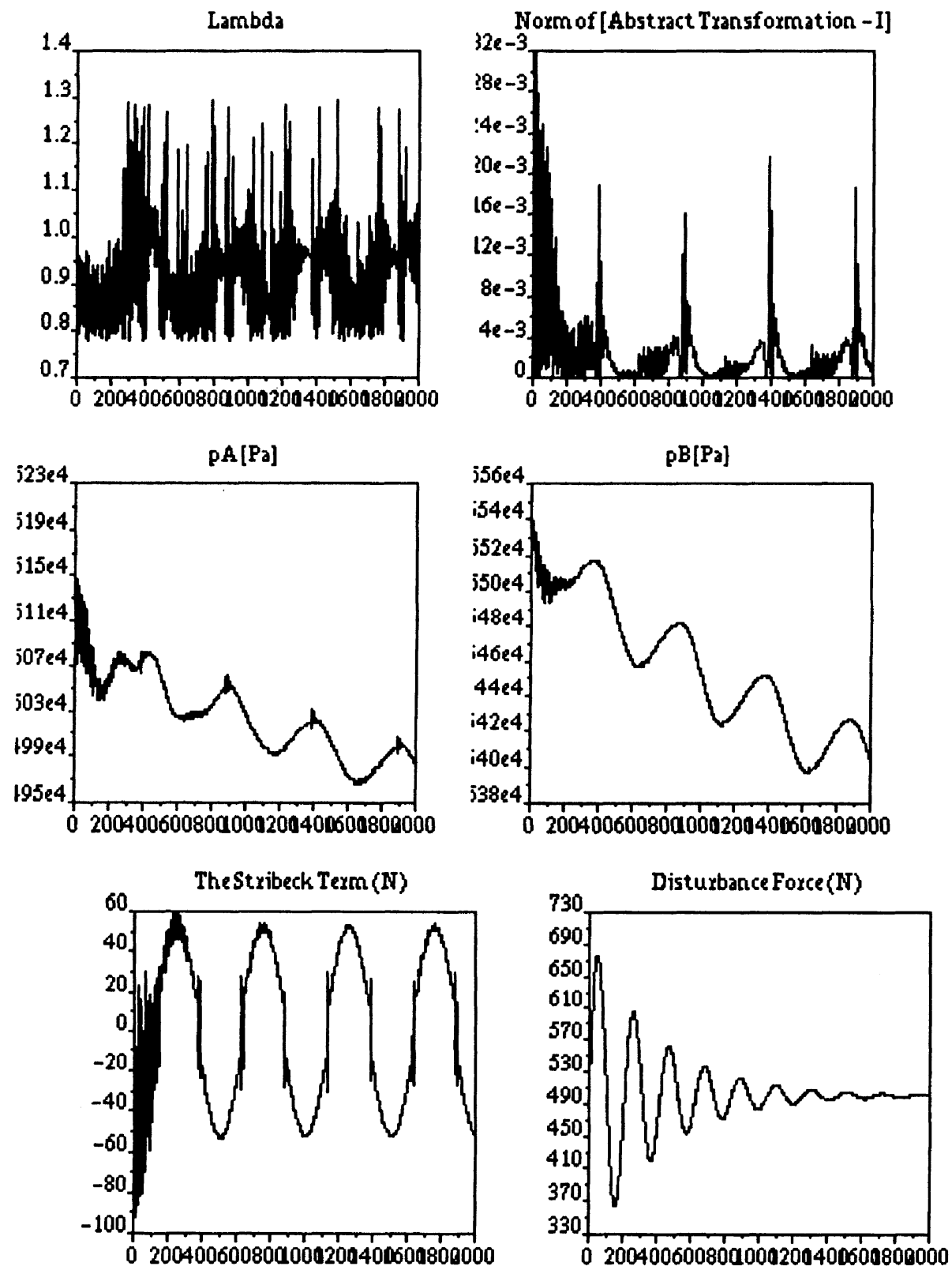

Figure 2 - The factor of the linear interpolation $(\lambda)$, the norm of the (T-I) matrices, the variation of the pressure in the chambers of the pistons $[P a]$, the friction force $[N]$ calculated according to the Stribeck model, and the external disturbance force $[N]$ are described.

At first the special symplectic matrices were applied for adaptation. In Fig. 1 the phase space, the trajectory of the nominal and the simulated motion, the trajectory tracking error, and the control signal of the hydraulic cylinder are described. It can well be seen from the figures that in spite of the considerable perturbations not taken into account in the rough model the adaptive control successfully learns the observable properties of the system and results in smooth variation of the pressure in the chambers of the cylinder. 

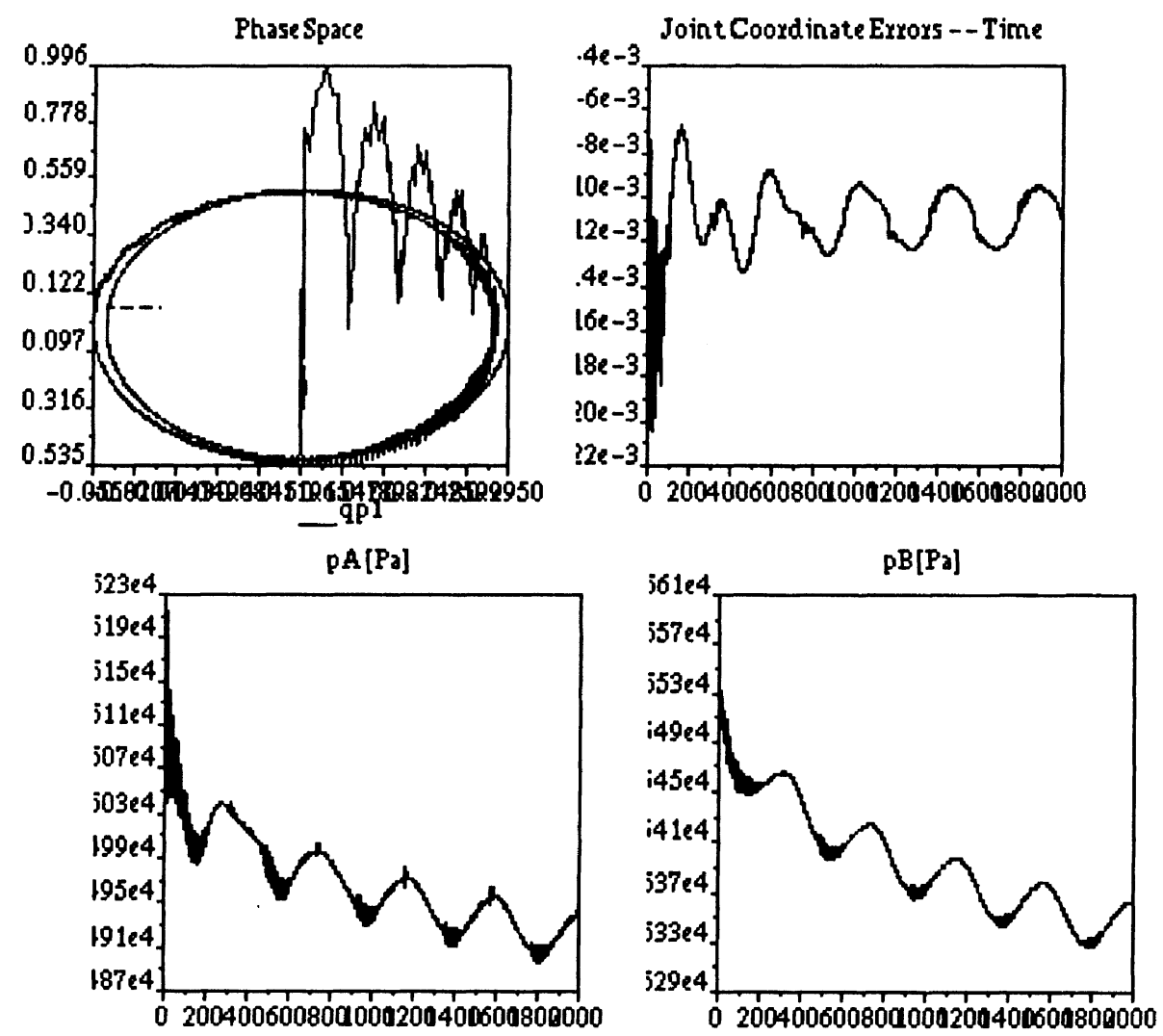

Figure 3 - The Counterparts of Certain Characteristics of the Symplectic Transformations Based Control in the Case of the Application of the Partially Stretched Orthogonal Transformations

In Fig. 3 the operation of the Partially Stretched Orthogonal Transformations based control are given for the same nominal trajectory and external perturbations. This latter approach seems to be a little bit more noisy. Fig. 4 reveals that the nonadaptive control under the same circumstances operates as a noisy bang-bang controller.
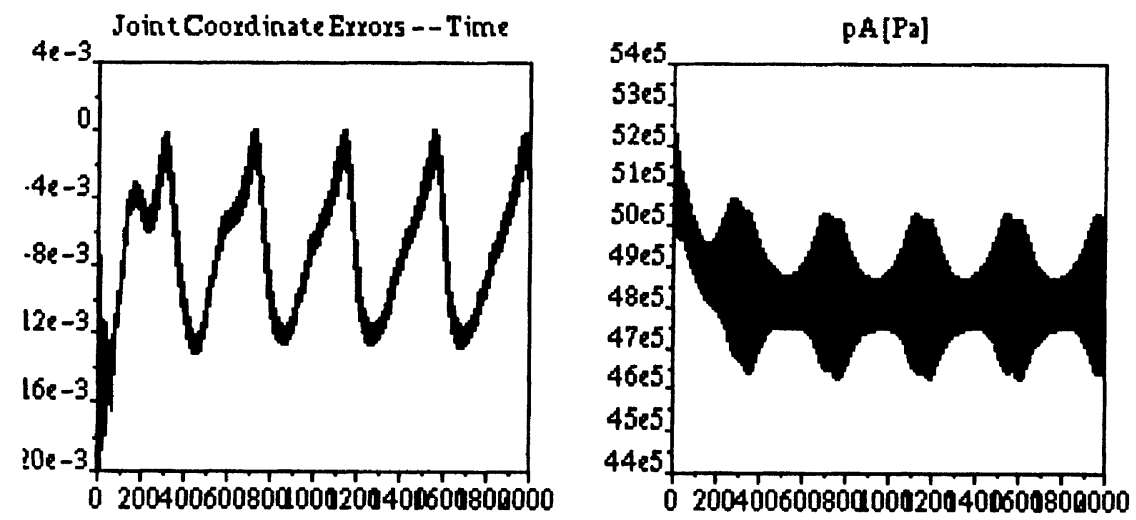

Figure 4 - The Behavior of the Non-Adaptive Control under the Same Circumstances 


\section{CONCLUSIONS}

In this paper a recently developed adaptive controller based on the "Modified Renormalization Algorithm", the "Partially Stretched Orthogonal Transformations", specially constructed "Symplectic Transformations", and a simple linear interpolation technique was applied to the control of a differential hydraulic cylinder under external disturbances not modeled by the controller. The simulation results well illustrate that such an adaptive technique can well improve the simple linear PI controller's operation. The information need of this adaptive controller is far much less than that of the traditional disturbance rejection controller needing the measurement of the disturbance force and its time-derivative. The simulation results well testify the fruitful co-operation of the linear interpolation part and the application of the symplectic / partially stretched orthogonal matrices. The method is promising in other fields of application where the behavior of a complex system under unknown environmental interaction is to be predicted: in appropriate column vectors several properties of time-dependent quantities $\left(0^{\text {th }}, 1^{\text {st }}, \mathrm{n}^{\text {th }}\right.$ order timederivatives) can be placed and mapped wit the convenient algebraic technique here presented.

\section{ACKNOWLEDGMENTS}

The authors thankfully acknowledge the support by the Hungarian National Research Fund in the program OTKA T 034651.

\section{REFERENCES}

1. Bröcker, M., Lemmen, M. Nonlinear Control Methods for Disturbance Rejection on a Hydraulically Driven Flexible Robot. Proc. of the 2nd Intl. Workshop On Robot Motion And Control, RoMoCo'001, October 18-20, 2001, Bukowy Dworek, Poland, pp. 213-217, ISBN: 83-7143-515-0, IEEE catalog Number: 01EX535.

2. Drakunov, S., Hanchin, G.D., Su, W.C. and Ozguner U. Nonlinear Control of a Rodless Pneumatic Servoactuator, or Sliding Modes versus Coulomb Friction, Automatica, 1997, Vol. 33, no. 7, pp. 1401-1408.

3. El Hini, Yahya. Comparison of the Application of the Symplectic and the Partially Stretched Orthogonal Transformations in a New Branch of Adaptive Control for Mechanical Devices. Proc. of the 10th Intl. Conference on Advanced Robotics, August 22-25, Budapest, Hungary, pp. 701 706, ISBN 9637154051.

4. Pátkai, B., Tar, J.K., Rudas, I.J., Bitó, J.F. Convergence Properties of the Modified Renormalization Algorithm Based Adaptive Control Supported by Ancillary Methods, under for publication in the 2002 IEEE Intl. Symp. on Industrial Electronics (ISIE 2002), July 8-11, 2002 L' Aquila, Italy.

5. Tar, J.K., Rontó M. Adaptive Control Based on the Application of Simplified Uniform Structures and Learning Procedures. Zbornik Radova, Vol. 24 No. 2, 2000, pp. 174-194. (ISSN: 0351-1804). 